\title{
High resolution functional photoacoustic computed tomography of the mouse brain during electrical stimulation
}

Mohammad R. N. Avanaki, Jun Xia, Lihong V. Wang

Mohammad R. N. Avanaki, Jun Xia, Lihong V. Wang, "High resolution functional photoacoustic computed tomography of the mouse brain during electrical stimulation ," Proc. SPIE 8581, Photons Plus Ultrasound: Imaging and Sensing 2013, 85813K (4 March 2013); doi: 10.1117/12.2004737

SPIE. Event: SPIE BiOS, 2013, San Francisco, California, United States 


\title{
High resolution functional photoacoustic computed tomography of the mouse brain during electrical stimulation
}

\author{
Mohammad R.N. Avanaki, Jun Xia, and Lihong V. Wang* \\ Optical Imaging Laboratory, Department of Biomedical Engineering, Washington University in St. Louis, St. Louis, MO 63130, USA \\ Corresponding author. Email: LHWANG@WUSTL.EDU
}

\begin{abstract}
Photoacoustic computed tomography (PACT) is an emerging imaging technique which is based on the acoustic detection of optical absorption from tissue chromophores, such as oxy-hemoglobin and deoxy-hemoglobin. An important application of PACT is functional brain imaging of small animals. The conversion of light to acoustic waves allows PACT to provide high resolution images of cortical vasculatures through the intact scalp. Here, PACT was utilized to study the activated areas of the mouse brain during forepaw and hindpaw stimulations. Temporal PACT images were acquired enabling computation of hemodynamic changes during stimulation. The stimulations were performed by trains of pulses at different stimulation currents (between 0.1 to $2 \mathrm{~mA}$ ) and pulse repetition rates (between $0.05 \mathrm{~Hz}$ to $0.01 \mathrm{~Hz}$ ). The response at somatosensory cortex-forelimb, and somatosensory cortex-hindlimb, were investigated. The Paxinos mouse brain atlas was used to confirm the activated regions. The study shows that PACT is a promising new technology that can be used to study brain functionality with high spatial resolution.
\end{abstract}

Keywords: Functional Imaging, Photoacoustic Tomography, Electrical Stimulation.

\section{INTRODUCTION}

Conventional neuroimaging modalities cannot be easily translated to small animals. For instance, functional magnetic resonance imaging (fMRI) requires a very high magnetic field in order to obtain a sufficient signal to noise ratio (SNR) and spatial resolution for small animal imaging [1,2]. Functional connectivity mapping with optical intrinsic signal imaging (fcOIS) was recently introduced as an alternative method to image functional connectivity in mice [3, 4]. In fcOIS, changes in local hemoglobin concentrations are determined based on changes in the reflected light intensity from the surface of the brain $[3,5]$. Therefore, neuronal activity can be measured through the neurovascular response, similar to the method used in fMRI. However, due to the diffusion of light in tissue, the spatial resolution of fcOIS is limited [3, 6], and the experiment has thus far been performed using an exposed skull preparation, which adds complexity for longitudinal imaging. Photoacoustic computed tomography (PACT) is an emerging imaging technique that is based on the acoustic detection of optical absorption from tissue chromophores, such as oxy-hemoglobin $\left(\mathrm{HbO}_{2}\right)$ and deoxyhemoglobin $(\mathrm{Hb})[7,8]$. This hybrid nature makes PACT capable of providing high resolution images of the brain while leaving the scalp intact $[9,10]$. In Figure 1, graphs of molar extinction coefficients of oxy $\left(\mathrm{HbO}_{2}\right)$ and deoxy $(\mathrm{Hb})$ hemoglobin for wavelengths ranging from $250 \mathrm{~nm}$ to $950 \mathrm{~nm}$ are shown.

In this paper, we utilize photoacoustic computed tomography imaging system to image the brain during paw stimulation. The experiment was performed using a $532 \mathrm{~nm}$ wavelength laser. The photoacoustic signal was therefore directly proportional to the total hemoglobin concentration $(\mathrm{HbT})$. In this study, we found that fore- and hind-paw stimulations resulted in specific activity in different subregions of the somatosensory cortex. We have used differential method to find activation maps. Thus, we expect that PACT can be developed into a powerful functional imaging modality for future brain research.

Photons Plus Ultrasound: Imaging and Sensing 2013, edited by Alexander A. Oraevsky, Lihong V. Wang, Proc. of SPIE Vol. 8581, 85813K - @ 2013 SPIE · CCC code: 1605-7422/13/\$18 - doi: 10.1117/12.2004737 


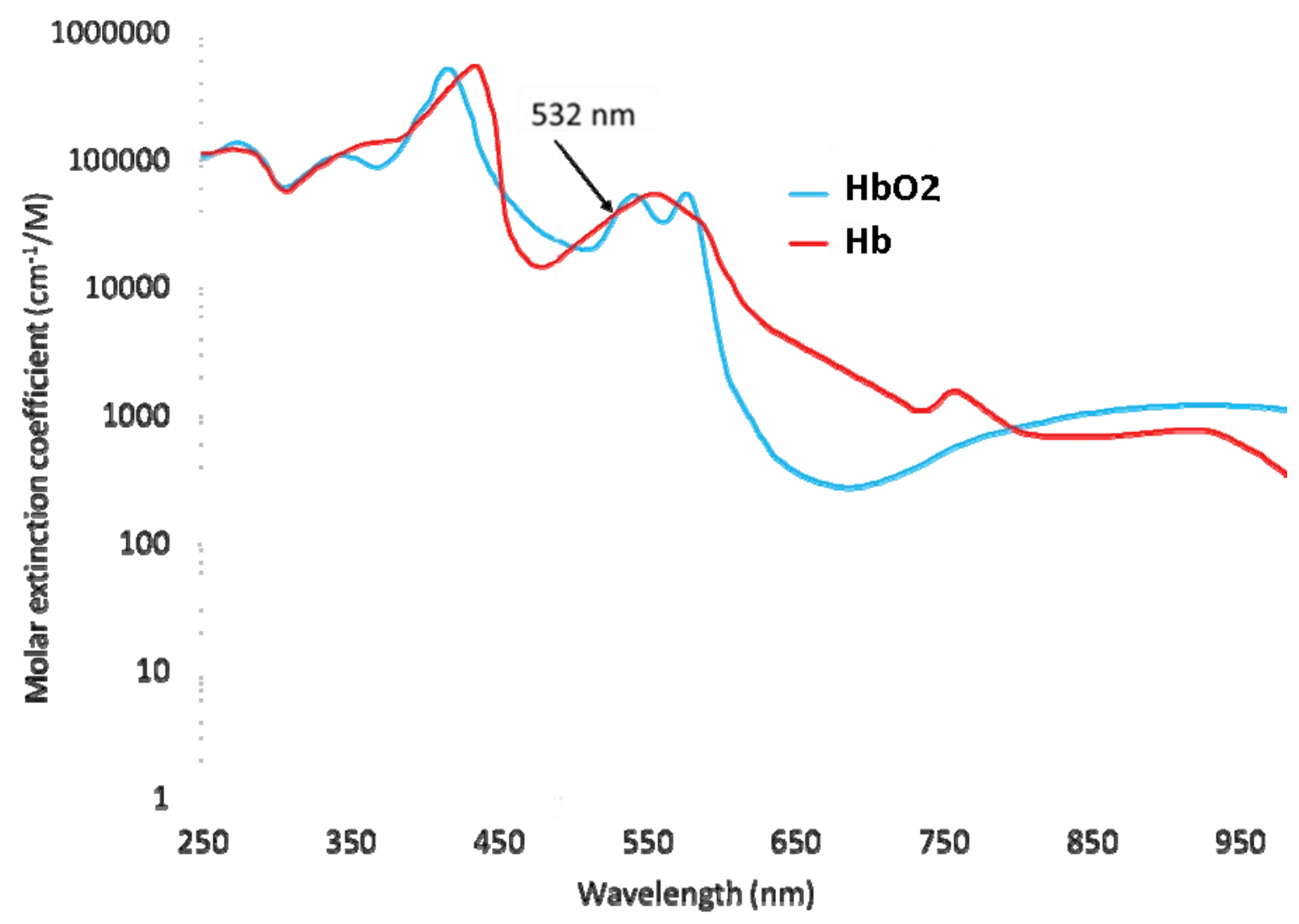

Figure 1. Graph showing molar extinction coefficients of oxy $\left(\mathrm{HbO}_{2}\right)$ and deoxy $(\mathrm{Hb})$ hemoglobin.

\section{IMAGING SETUP}

A schematic of the photoacoustic computed tomography system is shown in Figure 2. A Nd:YAG laser (Quantel, Brillant B) was the excitation source, at a pulse duration of 4-6 ns and a pulse repetition rate of $10 \mathrm{~Hz}$. The laser beam was homogenized by an optical diffuser, resulting in a $2 \mathrm{~cm}$ diameter beam on the surface of the mouse's head. We monitored the laser power fluctuation using a photodetector, and the data was recorded into the computer. The data was then used in the reconstruction algorithm. The maximum light intensity at the surface was approximately $15 \mathrm{~mJ} / \mathrm{cm}^{2}$, below the ANSI limit of $20 \mathrm{~mJ} / \mathrm{cm}^{2}$ at $532 \mathrm{~nm}$ wavelength which is the isosbestic point in the molar extinction coefficient graph showing in Figure 1 [11]. The resulting photoacoustic signals were detected by a $5 \mathrm{~cm}$ diameter, fullring ultrasonic transducer array (Imasonic Inc.) with 512 elements. The array had an $80 \%$ bandwidth at a central frequency of $5 \mathrm{MHz}$. Within the $2 \mathrm{~cm}$ diameter field of view, the system had an axial resolution of $100 \mu \mathrm{m}$, a lateral resolution of 100-200 $\mu \mathrm{m}$, and an elevational resolution of $1.0 \mathrm{~mm}$ [12].

The photoacoustic signal was digitalized by a 64-channel data acquisition system, with a full-ring acquisition taking 1.6 seconds [9]. For the experiments, we used 3-4 month old Swiss Webster mice. Before imaging, the animal was briefly anesthetized with $2 \%$ isoflurane, and the hair was removed with a hair remover lotion. The animal was then mounted on the imaging system. We changed the anesthetics from isoflurane to the mixture of ketamine and xylazine, because they provide stronger brain activity. $100 \mathrm{mg} / \mathrm{kg}$ ketamine and $10 \mathrm{mg} / \mathrm{kg}$ xylazine were mixed and administered intraperitoneally. For brain imaging, all experimental animal procedures were carried out in conformity with the guidelines of the US National Institutes of Health. The laboratory animal protocols for this work were in accordance with those approved by the Animal Studies Committee of Washington University in St. Louis. 


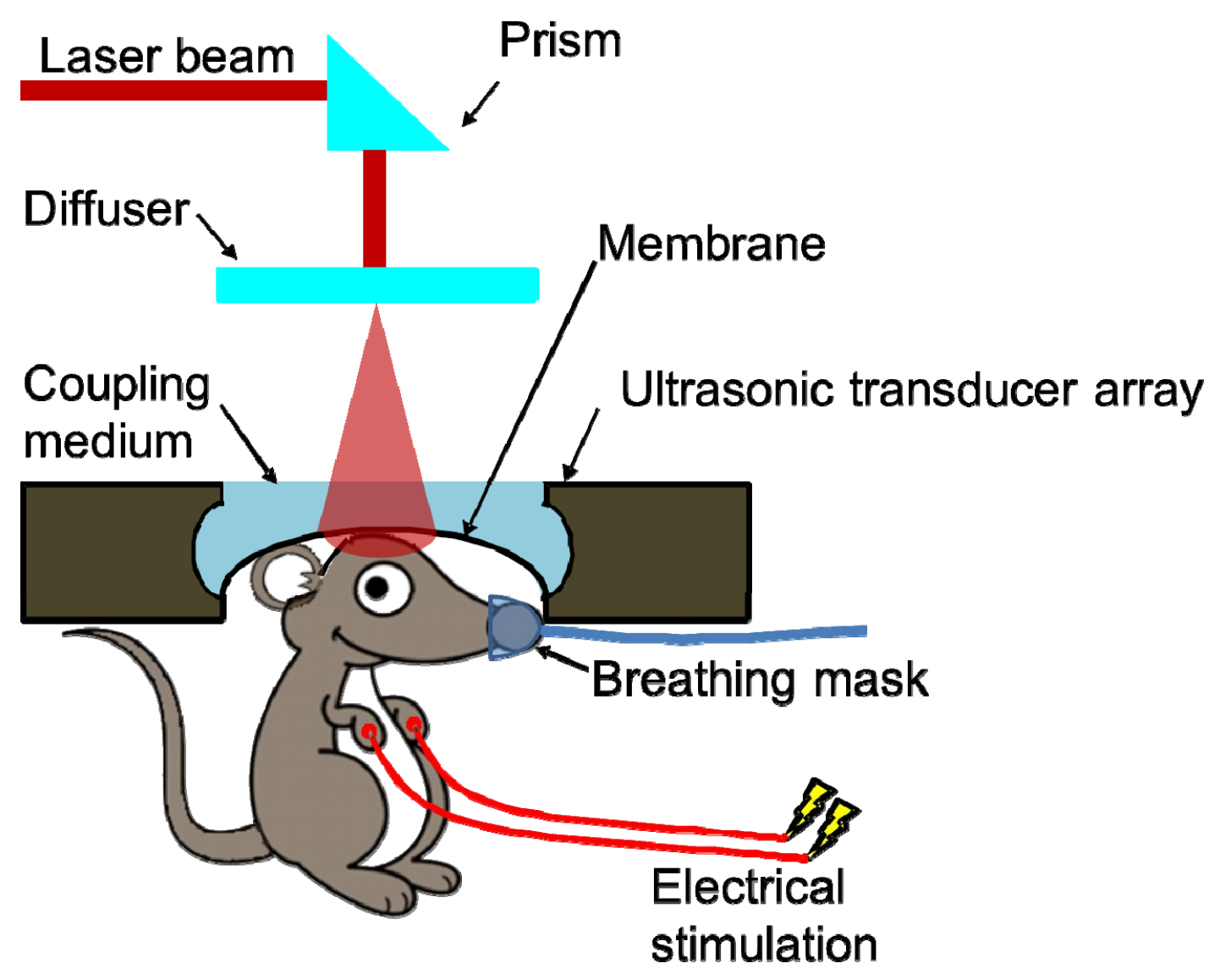

Figure 2. Schematic of the PACT system

\section{ATLAS PREPARATION}

To support the findings obtained from this study, a horizontal view atlas of the functional regions of the mouse brain was reconstructed from coronal view plates of the Paxinos atlas, which are $120 \mu \mathrm{m}$ apart [13]. Since the elevational resolution of the PACT system is about $1 \mathrm{~mm}$, extensions of the coronal view plates from the surface of the cortex down to $1 \mathrm{~mm}$ deep were considered for reconstruction. Because the plates are projected into one horizontal plate, we call the reconstructed atlas the averaged horizontal atlas.

There were eight main regions reconstructed in the atlas. These regions include the olfactory bulb, limbic, parietal, somatosensory, retrosplenial, visual, motor, and temporal regions. Also several subregions within the main regions were reconstructed in the atlas.

\section{STIMULATION PROCEDURE}

Prior to stimulation, the mouse was anesthetized by an intraperitoneal injection of $100 \mathrm{mg} / \mathrm{kg} \mathrm{ketamine} \mathrm{and} 10 \mathrm{mg} / \mathrm{kg}$ xylazine. Each of the four paws (left hindpaw, right hindpaw, left forepaw, and right forepaw) was stimulated in sequence using needle metallic electrodes inserted under the skin. The stimulation signal was pulsed with a 33\% duty cycle (i.e., $1 \mathrm{~mA}$ for $330 \mu \mathrm{s}$ and zero $\mathrm{mA}$ for $660 \mu \mathrm{s}$ ) at $1 \mathrm{~Hz}$ for 30 seconds, as shown in Figure 3. A 60 second recovery period was used between each set, and five such cycles were used for signal averaging. 
We chose to stimulate the paws since they are easily accessible and the animal is not required to perform any task. Paw stimulation activates the somatosensory region of the mouse brain due to the pain that the electrical stimulation generates.

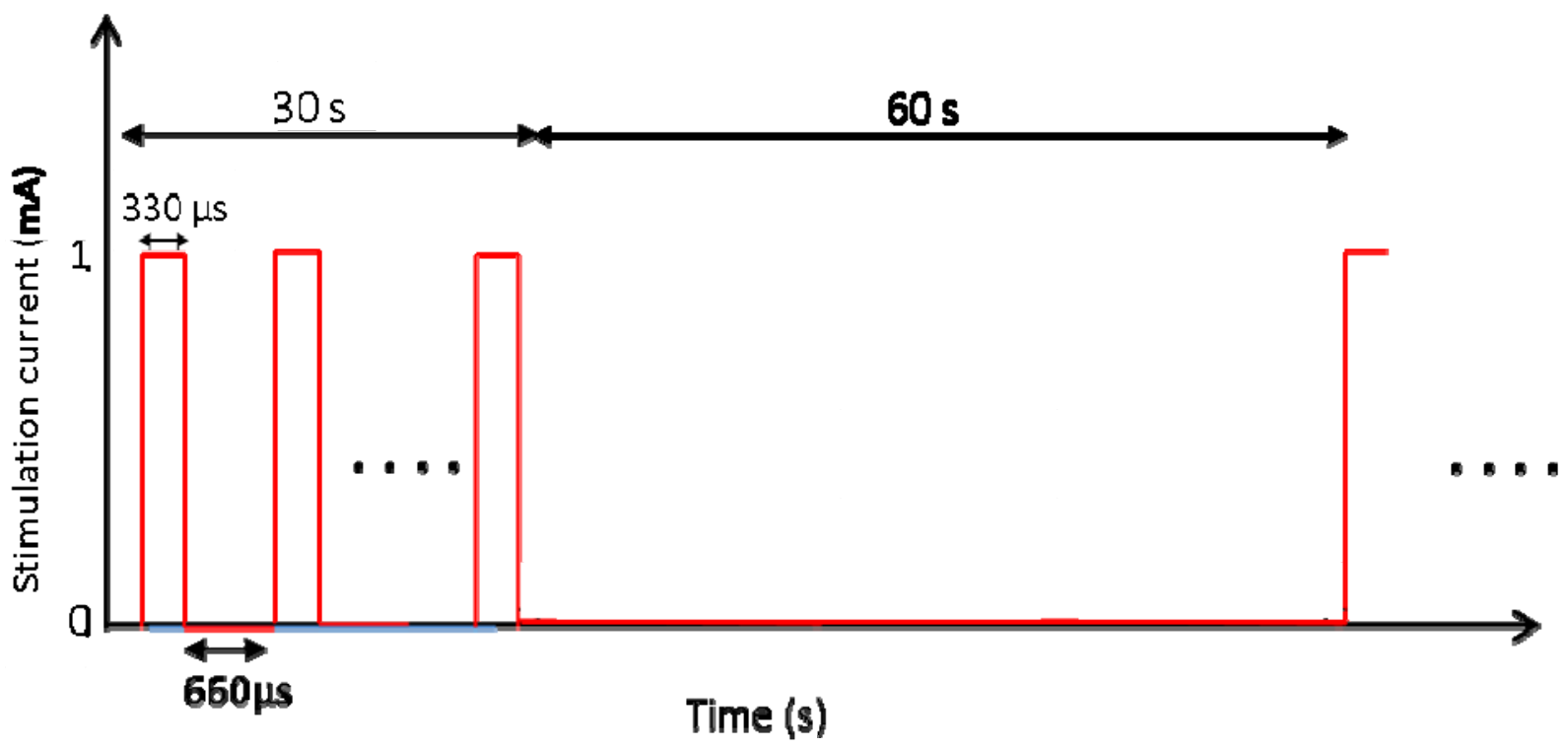

Figure 3. Paw electrical stimulation protocol.

\section{RESULTS}

We performed forepaw and hindpaw stimulation experiments to confirm the location of the somatosensory cortex and some of its sub-regions. Using the stimulation protocol, we stimulated the left hindpaw, the right hindpaw, the left forepaw, and the right forepaw in sequence, each for 6 minutes. During the stimulation process the paw electrical stimulation protocol was followed. The protocol is given in Figure 3.

The motion of the mouse head caused by the stimuli was corrected for in post processing. The post processing is as follows. The obtained images were first co-registered to the first image. The landmarks used for co-registration were the major blood vessels, including the inferior cerebral vein (between the olfactory bulb and frontal association), superior sagittal sinus (between the left and right hemispheres of the olfactory bulb, cingulate, and retrosplenial regions), transverse sinus (between the cerebellum and colliculi areas, and the visual and auditory cortices), and confluence of sinuses (intersection of the superior sagittal sinus and the transverse sinus).

Furthermore, the images were smoothed using a Gaussian filter. The Gaussian filter was created using "fspecial" built-in function in Matlab with two parameters hsize and sigma, where hsize is the size of the filter and sigma is the sigma of the

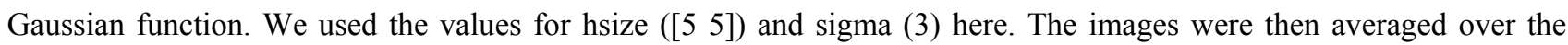
periods with stimuli $\left(I_{s}\right)$ and without stimuli $\left(I_{n s}\right)$, and a relative intensity image was computed pixel by pixel, using $\left(I_{s^{-}}\right.$ $\left.\mathrm{I}_{\mathrm{ns}}\right) / \mathrm{I}_{\mathrm{ns}}$. Figure 4 shows the activated areas in the somatosensory cortex induced by forepaw and hindpaw stimulations. The signals from the stimulated regions on the brain (left hindpaw, right hindpaw, left forepaw, and right forepaw) do not follow the exact shape of the stimulation signal given in Figure 3. This is due to the hemodynamic response function of the brain. Hemodynamic response function of the brain can be modeled and its parameters can be measured. This issue needs a comprehensive investigation in the future. 


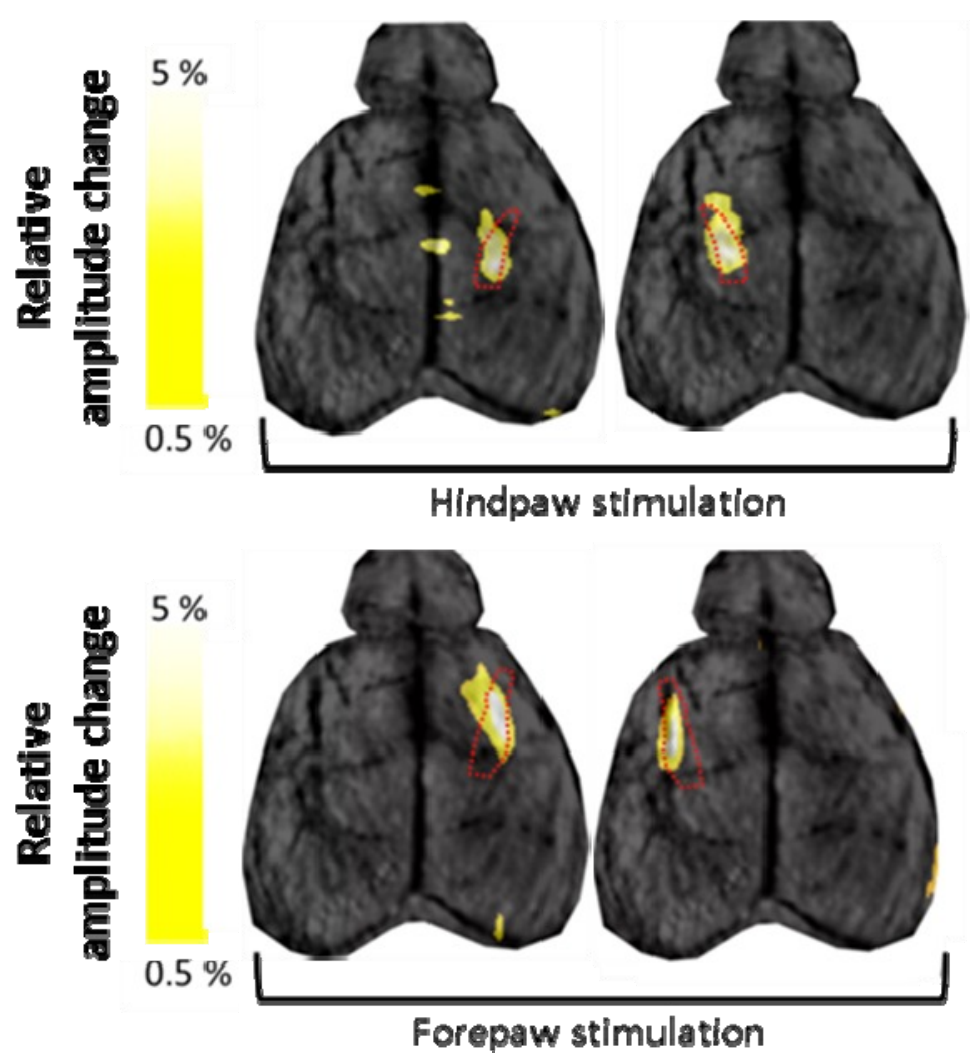

Figure 4. Hindpaw and forepaw stimulation results on a Swiss Webster mouse. The areas encircled by the dotted lines show the corresponding functional regions from the atlas.

\section{CONCLUSION}

Functional brain imaging of small animals has been a challenge for conventional neural imaging modalities. For instance, fMRI and fcOIS lack the spatial resolution to differentiate the functional sub-regions. Here, using photoacoustic computed tomography, the activated areas of the mouse brain during forepaw and hindpaw stimulation were studied with high spatial resolution. The response at four functional regions on the cortex, i.e, the somatosensory cortex-forelimb and somatosensory cortex-hindlimb in the left and right sides of the brain, were investigated. PACT has the unique advantage of directly sensing optical absorption with an acoustically defined high depth-to-resolution ratio, and we expect it will become a powerful functional imaging modality, allowing neurology researchers to study brain diseases in standardized mouse models.

\section{ACKNOWLEDGEMENTS}

The authors acknowledge and thank Prof. James Ballard, for his close look at the manuscript. This work was sponsored in part by National Institutes of Health grants R01 EB000712, R01 EB008085, R01 CA134539, R01 CA159959, U54 CA136398, R01 EB010049, R01 CA157277, and DP1 EB016986 (NIH Director's Pioneer Award). L.W. has a financial interest in Microphotoacoustics, Inc. and Endra, Inc., which, however, did not support this work.

\section{REFERENCES}

1. Benveniste, H. and S. Blackband, MR microscopy and high resolution small animal MRI: applications in neuroscience research. Progress in neurobiology, 2002. 67(5): p. 393-420. 
2. Jonckers, E., et al., Functional connectivity fMRI of the rodent brain: comparison of functional connectivity networks in rat and mouse. PloS one, 2011. 6(4): p. e18876.

3. White, B.R., et al., Imaging of functional connectivity in the mouse brain. PloS one, 2011. 6(1): p. e16322.

4. Bero, A.W., et al., Bidirectional relationship between functional connectivity and amyloid- $\beta$ deposition in mouse brain. The Journal of Neuroscience, 2012. 32(13): p. 4334-4340.

5. $\quad$ Bahar, S., et al., Intrinsic optical signal imaging of neocortical seizures: the'epileptic dip'. Neuroreport, 2006. 17(5): p. 499503.

6. Niu, H., et al., Resting-state functional connectivity assessed with two diffuse optical tomographic systems. Journal of biomedical optics, 2011. 16(4): p. 046006-046006-5.

7. Wang, L.V., Tutorial on photoacoustic microscopy and computed tomography. Selected Topics in Quantum Electronics, IEEE Journal of, 2008. 14(1): p. 171-179.

8. Yang, J.M., et al., Simultaneous functional photoacoustic and ultrasonic endoscopy of internal organs in vivo. Nature Medicine, 2012. 18(8): p. 1297-1302.

9. Gamelin, J., et al., A real-time photoacoustic tomography system for small animals. Optics express, 2009. 17(13): p. 1048910498 .

10. Yao, J., et al., Noninvasive photoacoustic computed tomography of mouse brain metabolism in vivo. Neuroimage, 2013. 64(0): p. 257-266.

11. Standard, A., Z136. 1. American national standard for the safe use of lasers. American National Standards Institute. Inc., New York, 1993.

12. Xia, J., et al., Three-dimensional photoacoustic tomography based on the focal-line concept. Journal of biomedical optics, 2011. 16(9): p. 090505-090505-3.

13. Paxinos, G. and K. Franklin, The mouse brain in stereotaxic coordinates: compact second edition. San Diego: Academic, 2003. 